\title{
Flato-Fronsdal theorem for higher-order singletons
}

\author{
Thomas Basile, ${ }^{a, b}$ Xavier Bekaert ${ }^{a}$ and Nicolas Boulanger ${ }^{b, 1}$ \\ ${ }^{a}$ Laboratoire de Mathématiques et Physique Théorique, \\ Unité Mixte de Recherche 7350 du CNRS, Fédération de Recherche 2964 Denis Poisson, \\ Université François Rabelais, Parc de Grandmont 37200 Tours, France \\ ${ }^{b}$ Service de Mécanique et Gravitation, Université de Mons - UMONS, \\ 20 Place du Parc, 7000 Mons, Belgique \\ E-mail: thomas.basile@umons.ac.be, xavier.bekaert@lmpt.univ-tours.fr, \\ nicolas.boulanger@umons.ac. be
}

ABSTRACT: We prove a generalized Flato-Fronsdal theorem for higher-order, scalar and spinor, singletons. In the resulting infinite tower of bulk higher-spin fields, we point out the occurrence of partially-massless fields of all depths. This leads us to conjecture a holographic duality between a higher-spin gravity theory around $A d S_{d+1}$ with the aforementioned spectrum of fields, and a free $C F T_{d}$ of fundamental (higher-order) scalar and spinor singletons. As a first check of this conjecture, we find that the total Casimir energy vanishes at one loop.

KeYwords: Higher Spin Symmetry, AdS-CFT Correspondence

ArXiv EPRINT: 1410.7668

À la mémoire de Francis A. Dolan

\footnotetext{
${ }^{1}$ Research Associate of the Fund for Scientific Research-FNRS (Belgium).
} 


\section{Contents}

1 Introduction 1

2 Notation and methodology 3

3 Characters of partially-massless fields and higher-order singletons $\quad 4$

3.1 Review of general results 4

3.2 Character of a generalised Verma module 5

$\begin{array}{lll}3.3 & \text { Characters of various specific highest-weight modules }\end{array}$

$\begin{array}{llr}4 & \text { Fusion rules for higher-order singletons } & 8\end{array}$

$\begin{array}{lll}5 & \text { Casimir energy } & 10\end{array}$

6 Conclusions and perspectives $\quad 12$

A General theorem 12

\section{Introduction}

The celebrated Flato-Fronsdal theorem [1] is at the very core of the kinematics underlying Vasiliev's four-dimensional nonlinear field equations for higher-spin gravity $[2,3] .{ }^{1}$ This theorem is also crucial for the higher-spin holographic conjectures [6-8] involving the free and critical 3-dimensional vector models of scalar and spinor fields living at the conformal infinity of $A d S_{4}$.

From the purely group-theoretical point of view, the Flato-Fronsdal theorem can be stated as the decomposition of the tensor product of two Dirac singletons [9] (either bosonic or fermionic) into an infinite direct sum of irreducible representations (irreps) of $\mathfrak{s o}(2,3)$. The latter representations can either be viewed as on-shell Fronsdal fields propagating in the bulk of $A d S_{4}$ or as conserved currents of the $C F T_{3}$. Correspondingly, the Dirac singletons (scalar and spinor) can be viewed as $A d S_{4}$ fields whose physical degrees of freedom live at its conformal infinity or as free conformal fields propagating in 3-dimensional Minkowski space. The infinite sum of $\mathfrak{s o}(2,3)$-irreps corresponds, from the bulk point of view, to an infinite tower of higher-spin gauge fields building up the spectrum of Vasiliev's theory. From the boundary point of view, the infinite sum corresponds to the single-trace sector of the corresponding vector models.

The Flato-Fronsdal theorem has been extended to higher-dimensional conformal algebras $\mathfrak{s o}(2, d)$ in [10-12]. In the work [11], the corresponding generalisations suggest the

\footnotetext{
${ }^{1}$ For a non-technical review explaining the key mechanisms at work in Vasiliev's 4D equations, see [4]. For a recent technical review, see e.g. [5].
} 
existence of extended (supersymmetric) nonlinear higher-spin theories in $A d S_{d+1}$, where mixed-symmetry fields, on top of the totally-symmetric ones, are propagating, together with some massive $p$-forms. In the latter work [12], the whole analysis is based on characters of $\mathfrak{s o}(2, d)$ and incorporates singletons of spin $s \geqslant 1$ (for $d$ even). Yet another class of generalisations features the so-called higher-order scalar singletons, ${ }^{2}$ that are non-unitary, ultra-short irreducible representations of the conformal algebra. The spectrum of $A d S_{d+1}$ fields appearing upon tensoring out higher-order scalar singletons comprises partially massless gauge fields of odd depths [14]. Totally-symmetric partially-massless fields were introduced in [15-17] (the frame-like formulation was given in [18]) and their holographic duals are partially-conserved currents [19]. Gauge fields always turned out to be of crucial importance, thereby it can be suspected that partially-massless field could be relevant in a unified framework for fundamental interactions, e.g. in a cosmological scenario [20] around $d S_{d+1}$ where they are unitary.

In [14], a version of higher-spin holography was proposed where a vector model at an isotropic Lifshitz fixed point is conjectured to be dual to a higher-spin gravity with spectrum given by a tower of massless and partially massless fields of unbounded integer spins - see also [21] for a review of the conjecture. As we will explain below, we are lead to extend this conjecture to spinor CFT's.

In the present note, we give a proof of the fusion rules presented in [14] using character formulae and generalise them to include higher-order spinor singletons. We actually compute the general case of the tensor product of two (scalar and spinor) singletons of different orders. A novelty in the case of the tensor product of two higher-order spinor singletons is that the resulting spectra contain even-depth partially-massless fields. The spectra also contain mixed-symmetry fields that are massless, partially-massless or massive. Somewhat less expected, we find extra degeneracies in the spectrum of massless and partially-massless (both symmetric and mixed-symmetric) fields. ${ }^{3}$ Along the lines of the holographic conjectures proposed in [8] and [14], we expect that the isotropic Liftshitz point of the Gross-Neveu model should be dual to a bulk theory containing the spectrum of fields found in the present paper.

If unbroken higher-spin gravity theories are indeed dual to free CFTs, they should therefore receive no quantum corrections. This is manifest in the non-standard off-shell proposal given in $[24,25]$. Several one-loop checks of this expectation were performed in [26-28] where the total vacuum energy was shown to cancel. As a first test of the validity of the holographic proposal we have made above with the isotropic Lifshitz fixed points, we find the cancellation of the total Casimir energy for all the spectra considered in the present note.

The paper is organised as follows: after we explain, in section 2, our methodology and notation, in section 3 we compute the $\mathfrak{s o}(2, d)$ characters of higher-order scalar and spinor singletons, as well as the characters of symmetric and mixed-symmetric fields, either (partially) massless or massive. The main tools employed in this section is a combination of

\footnotetext{
${ }^{2}$ Also called here multi-linetons in accordance with the terminology of [13] to which we refer for more details.

${ }^{3}$ Mixed-symmetry, partially-massless fields seem to have been first considered in [22, 23].
} 
the analyses presented in [12] and [29]. In section 4, we take the product of the characters of $\ell$ th-order scalar and spinor singletons found in the previous section and decompose the result into a sum of characters of indecomposable $\mathfrak{s o}(2, d)$-representations. The central result of our paper is given in a theorem that generalises the Flato-Fronsdal theorem in the way explained above. A corollary of our theorem is the computation, presented in section 5, of the total Casimir energy corresponding to the resulting spectra. We conclude in section 6 with some perspectives of future works. Finally, for the sake of completeness, in the appendix we give a more general result compared to our theorem presented in section 4 , in the sense that it concerns products of singletons (scalar and spinor) of distinct orders. The structure of the fusion rule is similar to the case presented in section 4 , but the depths of partially-massless fields run over different values and is more cumbersome to enunciate, which is the reason why we relegate that general result to the appendix. This general case can fit with a Vasiliev-like higher-spin gravity construction by considering the tensor product of a direct sum of distinct multi-linetons with itself. ${ }^{4}$

\section{Notation and methodology}

In this section, we spell out the approach that we followed in order to compute characters of $\mathfrak{s o}(2, d)$ and their fusion rules. We also present our notation. The computation of the characters of the highest-weight representations we are interested in proceeds following a three-step procedure:

(1) We use the method explained in [12] to compute the character of a Verma module. We then apply on it the $\mathfrak{s o}(d)$ Weyl group symmetrizer in order to obtain the character of the corresponding generalised Verma module; ${ }^{5}$

(2) The structure of possible (sub)singular modules of the resulting generalised Verma module is obtained upon inspection of the sequences given in the subsection 4.4. of [29]. It turns out that, for all the highest-weights we consider in detail, there is only one submodule which happens to be a generalised Verma module. The character of the latter is computed following the step 1;

(3) We finally subtract the characters obtained in the previous two steps in order to get the character of the corresponding irreducible module.

Notation. The rank of the orthogonal subalgebra $\mathfrak{s o}(d) \subset \mathfrak{s o}(2, d)$ is denoted by $r$, i.e. $D_{r} \cong \mathfrak{s o}_{\mathbb{C}}(2 r)$ and $B_{r} \cong \mathfrak{s o}_{\mathbb{C}}(2 r+1)$. In the present note, we will be only interested in $\mathfrak{s o}(2, d)$ representations with highest-weight $\Lambda=(-\Delta, \vec{s})$, where $\Delta$ is, in $A d S_{d+1} / C F T_{d}$ language, the scaling dimension of the corresponding primary operator or the minimal energy of the corresponding bulk field, and $\vec{s}=\left(s_{1}, \ldots, s_{r}\right)$ is a dominant-integral weight

\footnotetext{
${ }^{4}$ Note that higher tensor products of singletons were considered in [30, 31] and in the appendix E of [23] where the non-compositeness of a generic Metsaev [32] mixed-symmetry gauge field was shown.

${ }^{5}$ For more details on the notions of Verma modules and generalised Verma modules relevant to our discussion, see e.g. [13, 29].
} 
of $\mathfrak{s o}(d)$ in the orthonormal basis. More precisely,

$$
\begin{aligned}
& s_{1} \geqslant \ldots \geqslant s_{r} \geqslant 0 \quad \text { for } \quad d=2 r+1 \\
& s_{1} \geqslant \ldots \geqslant s_{r-1} \geqslant\left|s_{r}\right| \quad \text { for } \quad d=2 r,
\end{aligned}
$$

where the $s_{i}$ 's are either all integers or all half-integers. In the case where $d=2 r$, the Dirac spinor can be decomposed into left and right irreducible Weyl spinors $\left(\frac{1}{2}, \ldots, \frac{1}{2}, \frac{1}{2}\right)$ and $\left(\frac{1}{2}, \ldots, \frac{1}{2},-\frac{1}{2}\right)$, and it will be denoted $\frac{1}{2}$.

We denote by $\mathcal{V}(\Delta, \vec{s})$ (resp. $\mathcal{D}(\Delta, \vec{s})$ ) the generalised indecomposable (resp. irreducible) Verma module associated with the highest-weight $\Lambda=(-\Delta, \vec{s})$. The character of $\mathcal{V}(\Delta, \vec{s})$ is denoted by $\mathcal{A}_{[\Delta, \vec{s}]}^{(2, d)}\left(q, x_{1}, \ldots, x_{r}\right)$ while the character of $\mathcal{D}(\Delta, \vec{s})$ is denoted by $\chi_{[\Delta, \vec{s}]}^{(2, d)}\left(q, x_{1}, \ldots, x_{r}\right)$, where we used $x_{i}:=e^{\mu_{i}}$ with a generic weight $\vec{\mu}=\sum_{i=1}^{r} \mu_{i} \overrightarrow{\mathrm{e}}_{i}$ in the orthonormal basis for $\mathfrak{s o}(d)$. Similarly, $q:=e^{-\mathrm{e}_{0}}(\mu)=: e^{-\mu_{0}}$ where $\mu=\mu_{0} \mathrm{e}_{0}$ is a weight of $\mathfrak{s o}(2) \subset \mathfrak{s o}(2, d)$. The character of an irreducible representation of $\mathfrak{s o}(d)$ with dominantintegral weight $\vec{s}$ is denoted by $\chi_{\vec{s}}^{(d)}\left(x_{1}, \ldots, x_{r}\right)$. We use the following shortening notation for the following specific cases:

- $\left(s_{1}, \ldots, s_{j}, \boldsymbol{s}\right)$ is used to denote the following $\mathfrak{s o}(d)$-weight $\left(s_{1}, \ldots, s_{j}, s, \ldots, s\right)$ for which $s_{j+1}=s_{j+2}=\ldots=s_{r}=s$;

- In the previous rule, when several last components of a weight are all null, we will sometimes omit the symbol $\mathbf{0}$. For example $\left(s_{1}, \ldots, s_{j}, 0, \ldots, 0\right)$ will sometimes be denoted $\left(s_{1}, \ldots, s_{j}\right)$ instead of $\left(s_{1}, \ldots, s_{j}, \mathbf{0}\right)$, for the sake of brevity;

- The weights $(s_{1}, \ldots, s_{j}, \underbrace{1, \ldots, 1}_{m}, s_{j+m+1}, \ldots, s_{r})$ will be denoted $\left(s_{1}, \ldots, s_{j}, \mathbf{1}^{m}\right.$, $\left.s_{j+m+1}, \ldots, s_{r}\right)$.

\section{Characters of partially-massless fields and higher-order singletons}

In this section we first review some results of $[12,29]$ and use them to compute the characters of partially-massless fields and higher-order singletons.

\subsection{Review of general results}

We recall the following two results spelled out in [29]:

(1) For $\left(\mu_{1}, \ldots, \mu_{r}\right)$ a $\mathfrak{s o}(d)$ dominant-integral weight, and for

$-d=2 r+1$

If $\Delta$ is given by either $\Delta=r-\frac{1}{2}-n$ (for $n \in \mathbb{N}$ ) when $\mu_{1}, \ldots, \mu_{r}$ are all integers, or by $\Delta=r-n$ (for $n \in \mathbb{N}$ ) when $\mu_{1}, \ldots, \mu_{r}$ are all half-integers, then the irreducible module $\mathcal{D}\left(\Delta, \mu_{1}, \ldots, \mu_{r}\right)$ is given by the following quotient:

$$
\mathcal{D}\left(\Delta, \mu_{1}, \ldots, \mu_{r}\right) \cong \frac{\mathcal{V}\left(\Delta, \mu_{1}, \ldots, \mu_{r}\right)}{\mathcal{D}\left(d-\Delta, \mu_{1}, \ldots, \mu_{r}\right)},
$$


$-d=2 r$

If $\Delta$ is given by $\Delta=n-\mu_{n}$ for a given $n \in\{1,2, \ldots, r\}$, and obeys the conditions $\Delta \neq r$ and $-\Delta \pm \mu_{r}+r \in \mathbb{N}$, then the irreducible module $\mathcal{D}\left(\Delta, \mu_{1}, \ldots, \mu_{r}\right)$ is given by the same quotient as in (3.1).

In these two cases, $\mathcal{D}\left(d-\Delta, \mu_{1}, \ldots, \mu_{r}\right) \cong \mathcal{V}\left(d-\Delta, \mu_{1}, \ldots, \mu_{r}\right)$, meaning that there is no singular sub-module in $\mathcal{V}\left(d-\Delta, \mu_{1}, \ldots, \mu_{r}\right)$.

(2) For $\left(\lambda_{0}, \lambda_{1}, \ldots, \lambda_{r}\right)$ an $\mathfrak{s o}(d+2)$ dominant-integral weight, the irreducible module of highest-weight $\left(-\lambda_{1}-d+1, \lambda_{0}+1, \lambda_{2}, \ldots, \lambda_{r}\right)$ is given by:

$$
\mathcal{D}\left(\lambda_{1}+d-1, \lambda_{0}+1, \lambda_{2}, \ldots, \lambda_{r}\right) \cong \frac{\mathcal{V}\left(\lambda_{1}+d-1, \lambda_{0}+1, \lambda_{2}, \ldots, \lambda_{r}\right)}{\mathcal{D}\left(\lambda_{0}+d, \lambda_{1}, \lambda_{2}, \ldots, \lambda_{r}\right)}
$$

with $\mathcal{D}\left(\lambda_{0}+d, \lambda_{1}, \lambda_{2}, \ldots, \lambda_{r}\right) \cong \mathcal{V}\left(\lambda_{0}+d, \lambda_{1}, \lambda_{2}, \ldots, \lambda_{r}\right)$.

\subsection{Character of a generalised Verma module}

The character of an $\mathfrak{s o}(2, d)$ Verma module is given by [12]

$$
\mathcal{C}_{[\Delta, \vec{s}]}^{(d, 2)}\left(q, x_{1}, \ldots, x_{r}\right)=q^{\Delta} \mathcal{P}^{(d)}\left(q, x_{1}, \ldots, x_{r}\right) \mathcal{C}_{\vec{s}}^{(d)}\left(x_{1}, \ldots, x_{r}\right)
$$

where

$$
\mathcal{P}^{(d)}\left(q, x_{1}, \ldots, x_{r}\right)= \begin{cases}\frac{1}{(1-q)} \prod_{1 \leqslant i \leqslant r} \frac{1}{\left(1-q x_{i}\right)\left(1-q x_{i}^{-1}\right)} & \text { when } \quad d=2 r+1, \\ \prod_{1 \leqslant i \leqslant r} \frac{1}{\left(1-q x_{i}\right)\left(1-q x_{i}^{-1}\right)} & \text { when } \quad d=2 r\end{cases}
$$

and

$$
\mathcal{C}_{\vec{s}}^{(d)}\left(x_{1}, \ldots, x_{r}\right)=\left\{\begin{array}{lr}
\prod_{1 \leqslant i \leqslant r} x_{i}^{s_{i}+r+1 / 2-i}\left(x_{i}^{1 / 2}-x_{i}^{-1 / 2}\right) \Delta^{-1}\left(x_{1}+x_{1}^{-1}, \ldots, x_{r}+x_{r}^{-1}\right) \\
\prod_{1 \leqslant i \leqslant r} x_{i}^{s_{i}+r-i} \Delta^{-1}\left(x_{1}+x_{1}^{-1}, \ldots, x_{r}+x_{r}^{-1}\right) & \text { when } d=2 r+1,
\end{array}\right.
$$

for $\Delta\left(x_{1}, \ldots, x_{r}\right)=\prod_{1 \leqslant i<j \leqslant r}\left(x_{j}-x_{i}\right)$ the Vandermonde determinant. It turns out that the rational function $\mathcal{P}^{(d)}\left(q, x_{1}, \ldots, x_{r}\right)$ is invariant under the action of the Weyl group of $\mathfrak{s o}(d) .^{6}$

In order to obtain the character of the corresponding generalised Verma module, since the $\mathfrak{s o}(d)$ part of the initial weight is always taken to be dominant integral, it suffices to apply the Weyl-group symmetrizer of $\mathfrak{s o}(d)$ on $\mathcal{C}_{[\Delta, \vec{s}]}^{(d, 2)}\left(q, x_{1}, \ldots, x_{r}\right)$, resulting in the following formula for the character of the corresponding generalised Verma module:

$$
\mathcal{A}_{[\Delta, \vec{s}]}^{(d, 2)}\left(q, x_{1}, \ldots, x_{r}\right)=q^{\Delta} \mathcal{P}^{(d)}\left(q, x_{1}, \ldots, x_{r}\right) \chi_{\vec{s}}^{(d)}\left(x_{1}, \ldots, x_{r}\right),
$$

\footnotetext{
${ }^{6}$ The action of the Weyl group is reviewed e.g. in the appendix B of [12]. The Weyl group acts on the variables $x_{i}$ by permutations and changes of signs.
} 
where, introducing

$$
k_{i}:=s_{i}+r+\frac{1}{2}-i
$$

the irreducible characters of $\mathfrak{s o}(d)$ are

$$
\chi_{\vec{s}}^{(d)}\left(x_{1}, \ldots, x_{r}\right)=\left\{\begin{array}{c}
\operatorname{det}\left(x_{i}^{k_{j}}-x_{i}^{-k_{j}}\right) \Delta^{-1}\left(x_{1}+x_{1}^{-1}, \ldots, x_{r}+x_{r}^{-1}\right) \prod_{1 \leqslant i \leqslant r}\left(x_{i}^{1 / 2}-x_{i}^{-1 / 2}\right) \\
\text { when } d=2 r+1, \quad \text { and } \\
\frac{1}{2}\left(\operatorname{det}\left(x_{i}^{k_{j}}-x_{i}^{-k_{j}}\right)+\operatorname{det}\left(x_{i}^{k_{j}}+x_{i}^{-k_{j}}\right)\right) \Delta^{-1}\left(x_{1}+x_{1}^{-1}, \ldots, x_{r}+x_{r}^{-1}\right) \\
\text { when } \quad d=2 r .
\end{array}\right.
$$

\subsection{Characters of various specific highest-weight modules}

Multi-linetons Di and Rac. Analogously to the terminology of Flato and Fronsdal, we call Di (resp. Rac) $\ell$-lineton the irreducible $\mathfrak{s o}(2, d)$ module given by $\ell$ semi-infinite lines in compact weight space based on the lowest-energy states $\left|\frac{d+1}{2}-\ell, \frac{1}{2}\right\rangle$ (resp. the state $\left|\frac{d}{2}-\ell, \mathbf{0}\right\rangle$ ). Indeed, for $\ell=1$ and $d=3$ they correspond to the Di (resp. Rac) singleton of Dirac [1].

Using the results summarised in the subsection 3.1, item (1), one finds that the irreducible modules corresponding to the Rac and Di $\ell$-linetons are given in terms of the following quotients:

$$
\mathcal{D}\left(\frac{d}{2}-\ell, \mathbf{0}\right) \cong \frac{\mathcal{V}\left(\frac{d}{2}-\ell, \mathbf{0}\right)}{\mathcal{D}\left(\frac{d}{2}+\ell, \mathbf{0}\right)}
$$

and

$$
\mathcal{D}\left(\frac{d+1}{2}-\ell, \frac{\mathbf{1}}{\mathbf{2}}\right) \cong \frac{\mathcal{V}\left(\frac{d+1}{2}-\ell, \frac{\mathbf{1}}{\mathbf{2}}\right)}{\mathcal{D}\left(\frac{d-1}{2}+\ell, \frac{\mathbf{1}}{\mathbf{2}}\right)}
$$

with $\mathcal{D}\left(\ell+\frac{d}{2}, \mathbf{0}\right) \cong \mathcal{V}\left(\ell+\frac{d}{2}, \mathbf{0}\right)$ and $\mathcal{D}\left(\ell+\frac{d-1}{2}, \frac{1}{2}\right) \cong \mathcal{V}\left(\ell+\frac{d-1}{2}, \frac{1}{2}\right)$. The characters of the corresponding modules are

$$
\begin{aligned}
\chi_{\left[\frac{d}{2}-\ell, 0\right]}^{(d, 2)}\left(q, x_{1}, \ldots, x_{r}\right) & =q^{\frac{d}{2}}\left(q^{-\ell}-q^{\ell}\right) \mathcal{P}^{(d)}\left(q, x_{1}, \ldots, x_{r}\right), \\
\chi_{\left[\frac{d+1}{2}-\ell, \frac{1}{2}\right]}^{(d, 2)}\left(q, x_{1}, \ldots, x_{r}\right) & =q^{\frac{d}{2}}\left(q^{-\left(\ell-\frac{1}{2}\right)}-q^{\ell-\frac{1}{2}}\right) \chi_{\frac{1}{2}}^{(d)}\left(x_{1}, \ldots, x_{r}\right) \mathcal{P}^{(d)}\left(q, x_{1}, \ldots, x_{r}\right) .
\end{aligned}
$$

In the case $d=2 r$, there is a restriction on $\ell$ for the modules to be irreducible. It turns out that, for the scalar case, irreducibility implies $\ell<r$ and, for the spinor case, $\ell \leqslant r$. This being said, when $\ell$ does not satisfy these inequalities, the corresponding highest-weights are dominant integral. In order to make the latter modules irreducible, one has to further quotient them and the resulting irreducible modules happen to be finitedimensional. They correspond to $\mathfrak{s o}(2, d)$ spherical harmonics, e.g. of spin (or degree) $\ell-r \geqslant 0$ for the scalar case $\mathcal{D}(r-\ell, 0)$. These irreducible subspaces of solutions of the higher-order D'Alembertian equations will not be of interest to us since they are finitedimensional. We will slightly abuse the notation and still use the symbols $\mathcal{D}\left(\frac{d}{2}-\ell, \mathbf{0}\right)$ and $\mathcal{D}\left(\frac{d+1}{2}-\ell, \frac{1}{2}\right)$ for the corresponding reducible quotient modules (3.9) and (3.10). 
Bulk scalar fields. The structure of the module corresponding to a generic scalar field is $\mathcal{D}(\Delta, \mathbf{0}) \cong \mathcal{V}(\Delta, \mathbf{0})$. The character for a scalar field propagating in the bulk of $A d S_{d+1}$ therefore is

$$
\mathcal{A}_{[\Delta, \mathbf{0}]}^{(d, 2)}\left(q, x_{1}, \ldots, x_{r}\right)=q^{\Delta} \mathcal{P}^{(d)}\left(q, x_{1}, \ldots, x_{r}\right) .
$$

The unitarity condition is $\Delta \geqslant \frac{d-2}{2}$. The fields are reducible when $\Delta=\frac{d-2 \ell}{2}$, for $\ell \in \mathbb{N}_{0}$, and for these values of the conformal weight, one is back to the cases of the higher-order scalar singletons described above.

With a slight abuse of language inherited from the philosophy in higher-spin gravity, the irreps $\mathcal{D}(d-1-t, \mathbf{0}) \cong \mathcal{V}(d-1-t, \mathbf{0})$ will be called massless for $t=1$ and partially massless for $t>1$. This is justified by the fact that their scaling dimension $\Delta$ coincides with the scaling dimension of the corresponding spin-s irreps when $s=0$. However, as $\mathfrak{s o}(2, d)$ irreducible modules, they have a different structure compared to the (partially) massless modules in the sense that they do not arise from a quotient of generalized Verma modules. Actually, this is not totally correct in even dimensions $d=2 r$, where there exist values of $t$ for which $\mathcal{V}(d-1-t, \mathbf{0})$ admits singular sub-modules, so that the isomorphism $\mathcal{D}(d-1-t, \mathbf{0}) \cong \mathcal{V}(d-1-t, \mathbf{0})$ is not true. This arises when $2 r-1-t=r-\ell \Leftrightarrow t=r-1+\ell$ for $\ell \geqslant 1$.

Totally-symmetric fields/operators. Using the results summarised in the subsection 3.1, item (2), the structure of the modules corresponding to (partially-)massless, totally-symmetric fields/operators is

$$
\mathcal{D}(s+d-t-1, s) \cong \frac{\mathcal{V}(s+d-t-1, s)}{\mathcal{D}(s+d-1, s-t)}, \quad \text { for } \quad 1 \leqslant t \leqslant s
$$

where $\mathcal{D}(s+d-1, s-t) \cong \mathcal{V}(s+d-1, s-t)$, whence the character:

$$
\begin{array}{lr}
\mathcal{A}_{[s+d-t-1, s]}^{(d, 2)}\left(q, x_{1}, \ldots, x_{r}\right)=q^{s+d-t-1} \chi_{(s)}^{(d)}\left(x_{1}, \ldots, x_{r}\right) \mathcal{P}^{(d)}\left(q, x_{1}, \ldots, x_{r}\right) & \text { when } t>s \\
\chi_{[s+d-t-1, s]}^{(d, 2)}\left(q, x_{1}, \ldots, x_{r}\right)=q^{s+d-1}\left(q^{-t} \chi_{(s)}^{(d)}-\chi_{(s-t)}^{(d)}\right) \mathcal{P}^{(d)}\left(q, x_{1}, \ldots, x_{r}\right) & \text { when } t \leqslant s .
\end{array}
$$

Totally-symmetric, spin- $s$, massless fields/conserved currents correspond to $t=1$. Totallysymmetric, depth- $t$, partially-massless fields/partially-conserved currents correspond to $2 \leqslant$ $t \leqslant s$. Totally-symmetric, massive fields/operators correspond to $t>s$.

The same situation occurs for the modules $\mathcal{V}\left(s+d-t-1, s, \frac{1}{2}\right), s$ half-integer, $t \in \mathbb{N}_{0}$, that possess one sub-module $\mathcal{D}\left(s+d-1, s-t, \frac{\mathbf{1}}{\mathbf{2}}\right) \cong \mathcal{V}\left(s+d-1, s-t, \frac{\mathbf{1}}{\mathbf{2}}\right)$ for $s \geqslant t+1 / 2$. We thus have:

$$
\mathcal{D}\left(s+d-t-1, s, \frac{\mathbf{1}}{\mathbf{2}}\right) \cong \frac{\mathcal{V}\left(s+d-t-1, s, \frac{\mathbf{1}}{\mathbf{2}}\right)}{\mathcal{D}\left(s+d-1, s-t, \frac{\mathbf{1}}{\mathbf{2}}\right)}
$$


and the corresponding characters

$$
\begin{aligned}
& \mathcal{A}_{\left[s+d-t-1, s, \frac{1}{2}\right]}^{(d, 2)}\left(q, x_{1}, \ldots, x_{r}\right)=q^{s+d-t-1} \chi_{\left(s, \frac{1}{2}\right)}^{(d)}\left(x_{1}, \ldots, x_{r}\right) \mathcal{P}^{(d)}\left(q, x_{1}, \ldots, x_{r}\right) \\
& \text { when } t>s-1 / 2 \\
& \chi_{\left[s+d-t-1, s, \frac{1}{2}\right]}^{(d, 2)}\left(q, x_{1}, \ldots, x_{r}\right)=q^{s+d-1}\left(q^{-t} \chi_{\left(s, \frac{1}{2}\right)}^{(d)}-\chi_{\left(s-t, \frac{1}{2}\right)}^{(d)}\right) \mathcal{P}^{(d)}\left(q, x_{1}, \ldots, x_{r}\right) \\
& \text { when } t \leqslant s-1 / 2 \text {. }
\end{aligned}
$$

Mixed-symmetry fields/operators. Similarly, the modules with highest-weights $(t+$ $\left.1-s-d, s, \mathbf{1}^{m}\right)$ with $s, t \in \mathbb{N}_{0}$, possess one sub-module with highest-weight $\left(1-s-d, s-t, \mathbf{1}^{m}\right)$ for $s>t$. We thus have:

$$
\mathcal{D}\left(s+d-t-1, s, \mathbf{1}^{m}\right) \cong \frac{\mathcal{V}\left(s+d-t-1, s, \mathbf{1}^{m}\right)}{\mathcal{D}\left(s+d-1, s-t, \mathbf{1}^{m}\right)}
$$

with $\mathcal{D}\left(s+d-1, s-t, \mathbf{1}^{m}\right) \cong \mathcal{V}\left(s+d-1, s-t, \mathbf{1}^{m}\right)$. The characters are

$$
\begin{array}{r}
\mathcal{A}_{\left[s+d-t, s+1, \mathbf{1}^{m}\right]}^{(d, 2)}\left(q, x_{1}, \ldots, x_{r}\right)=q^{s+d-t} \chi_{\left(s+1, \mathbf{1}^{m}\right)}^{(d)}\left(x_{1}, \ldots, x_{r}\right) \mathcal{P}^{(d)}\left(q, x_{1}, \ldots, x_{r}\right) \\
\chi_{\left[s+d-t, s+1, \mathbf{1}^{m}\right]}^{(d, 2)}\left(q, x_{1}, \ldots, x_{r}\right)=q^{s+d}\left(q^{-t} \chi_{\left(s+1, \mathbf{1}^{m}\right)}^{(d)}-\chi_{\left(s-t+1, \mathbf{1}^{m}\right)}^{(d)}\right) \mathcal{P}^{(d)}\left(q, x_{1}, \ldots, x_{r}\right) \\
\text { when } t<s .
\end{array}
$$

Hook-shaped, spin-s, massless fields/conserved currents correspond to $t=1 .^{7}$ Hookshaped, depth- $t$, partially-massless fields or partially-conserved currents correspond to $2 \leqslant$ $t \leqslant s-1$. Hook-shaped, massive fields/operator correspond to $t \geqslant s$.

In the previous three cases, namely the totally-symmetric fields (bosonic and fermionic) and the mixed-symmetric ones, the massive modules can, in some sporadic cases, become reducible so that we abuse the notation when using the symbols $\mathcal{A}$ and $\mathcal{D}$. For example, in the totally-symmetric, massive spin- $s$ cases, when $t>s$ and for $d=2 r$, there are sporadic cases when the modules are reducible, in which cases we will abuse the notation when we will write $\mathcal{D}(s+d-1, s-t)$ instead of $\mathcal{V}(s+d-1, s-t)$.

Unitarity. The modules $\mathcal{D}(s+d-t-1, s), \mathcal{D}\left(s+d-t-1, s, \frac{\mathbf{1}}{\mathbf{2}}\right)$ and $\mathcal{D}\left(s+d-t, s, \mathbf{1}^{m}\right)$ are unitary only in the case $t=1$, as are the scalar and spinor $\ell$-linetons $\mathcal{D}\left(\frac{d}{2}-\ell, \mathbf{0}\right)$ and $\mathcal{D}\left(\frac{d+1}{2}-\ell, \frac{1}{2}\right)$ only in the case $\ell=1$.

\section{Fusion rules for higher-order singletons}

We now give the main result of the present note.

Theorem. The tensor product of two $\ell$-linetons, scalar and spinor, decomposes as:

$$
\begin{aligned}
& \mathcal{D}\left(\frac{d}{2}-\ell, \mathbf{0}\right) \otimes \mathcal{D}\left(\frac{d}{2}-\ell, \mathbf{0}\right) \cong \bigoplus_{k=1}^{\ell} \bigoplus_{s=0}^{\infty} \mathcal{D}(s+d-2 k, s, \mathbf{0}), \\
& \mathcal{D}\left(\frac{d+1}{2}-\ell, \frac{\mathbf{1}}{\mathbf{2}}\right) \otimes \mathcal{D}\left(\frac{d}{2}-\ell, \mathbf{0}\right) \cong \bigoplus_{t=1}^{2 \ell-1} \bigoplus_{s=\frac{1}{2}, \frac{3}{2}, \ldots}^{\infty} \mathcal{D}\left(s+d-t-1, s, \frac{\mathbf{1}}{\mathbf{2}}\right) .
\end{aligned}
$$

\footnotetext{
${ }^{7}$ The mixed-symmetry gauge fields corresponding to $t=1$ were first discussed by Metsaev in [32].
} 
In the cases $d=2 r+1$, we have

$$
\begin{aligned}
& \mathcal{D}\left(\frac{d+1}{2}-\ell, \frac{\mathbf{1}}{\mathbf{2}}\right) \otimes \mathcal{D}\left(\frac{d+1}{2}-\ell, \frac{\mathbf{1}}{\mathbf{2}}\right) \\
& \cong \bigoplus_{t=2-2 \ell}^{2 \ell-2} \mathcal{D}(d-1-t, \mathbf{0}) \oplus \bigoplus_{s=1}^{\infty} \bigoplus_{m=0}^{r-1} \mathcal{D}\left(s+d-2 \ell, s, \mathbf{1}^{m}\right) \\
& \quad \oplus \bigoplus_{t=1}^{2 \ell-2} \bigoplus_{s=1}^{\infty} \bigoplus_{m=0}^{r-1} 2 \mathcal{D}\left(s+d-t-1, s, \mathbf{1}^{m}\right),
\end{aligned}
$$

while in the cases $d=2 r$,

$$
\begin{aligned}
& \mathcal{D}\left(\frac{d+1}{2}-\ell, \frac{\mathbf{1}}{\mathbf{2}}\right) \otimes \mathcal{D}\left(\frac{d+1}{2}-\ell, \frac{\mathbf{1}}{\mathbf{2}}\right) \cong \bigoplus_{t=2-2 \ell}^{2 \ell-2} 2 \mathcal{D}(d-1-t, \mathbf{0}) \oplus \\
& \oplus \bigoplus_{s=1}^{\infty} \bigoplus_{m=0}^{r-2} 2 \mathcal{D}\left(s+d-2 \ell, s, \mathbf{1}^{m}\right) \\
& \oplus \bigoplus_{s=1}^{\infty}\left[\mathcal{D}\left(s+d-2 \ell, s, \mathbf{1}^{r-2}, 1\right) \oplus \mathcal{D}\left(s+d-2 \ell, s, \mathbf{1}^{r-2},-1\right)\right] \\
& \oplus \bigoplus_{t=1}^{2 \ell-2} \bigoplus_{s=1}^{\infty} \bigoplus_{m=0}^{r-2} 4 \mathcal{D}\left(s+d-t-1, s, \mathbf{1}^{m}\right) \\
& \oplus \bigoplus_{t=1}^{2 \ell-2} \bigoplus_{s=1}^{\infty}\left[2 \mathcal{D}\left(s+d-t-1, s, \mathbf{1}^{r-2}, 1\right) \oplus 2 \mathcal{D}\left(s+d-t-1, s, \mathbf{1}^{r-2},-1\right)\right]
\end{aligned}
$$

We interpret these 3 decompositions as follows.

- In the decomposition (4.1), the tensor product of two Rac $\ell$-lineton gives [14] a sum over all integer-spin, partially massless, totally-symmetric fields of odd depths $t$ going from $t=1$ to $t=2 \ell-1$, where strictly speaking the cases $t=1$ correspond to the massless fields and the cases $t>s$ correspond to massive fields;

- In the decomposition (4.2), the tensor product of a Di $\ell$-lineton with a Rac $\ell$-lineton gives a sum over all half-integer-spin, partially massless, totally-symmetric fields of all depths $t$ going from $t=1$ to $t=2 \ell-1$;

- In the decomposition (4.3), three types of fields appear:

- a finite multiplet of scalar fields, among which we find $2 \ell-1$ massive fields $\mathcal{D}(d-1-t, \mathbf{0})$ for $2-2 \ell \leqslant t \leqslant 0$ and $2 \ell-2$ partially massless for $0<t \leqslant 2 \ell-2$;

- a finite collection $\{\mathcal{D}(s+d-2 \ell, s, \mathbf{0})\}_{s=1,2, \ldots, 2 \ell-2}$ of massive spin-s fields;

- an infinite collection $\{\mathcal{D}(s+d-2 \ell, s, \mathbf{0})\}_{s=2 \ell-1, \ldots}$ of partially-massless spin-s fields of depth $t=2 \ell-1$;

- a finite collection $\left\{\mathcal{D}\left(s+d-2 \ell, s, \mathbf{1}^{m}\right)\right\}_{s=1,2, \ldots, 2 \ell-1 ; m=1, \ldots, r-1}$ of mixedsymmetry (hook) massive fields; 
- an infinite collection $\left\{\mathcal{D}\left(s+d-2 \ell, s, \mathbf{1}^{m}\right)\right\}_{s=2 \ell, \ldots ; m=1, \ldots, r-1}$ of partiallymassless mixed-symmetry (hook) fields of depth $t=2 \ell-1$; and

- a multiplicity-two tower of fields containing massive, partially-massless and massless fields, either totally symmetric $(m=0)$ or hook-shaped $(m>0)$ of various depths below $2 \ell-2$.

- In the even-dimensional case the product of two Di $\ell$-linetons (4.4) differs from the odd-dimensional case (4.3) by the overall multiplicity of two, apart from the hooklike mixed-symmetry fields for which the first column has maximal height $r$. We can track back the origin of this multiplicity by remembering that we actually consider the tensor product of two Dirac, not Weyl, $\ell$-linetons. The product of two left (resp. right) $\ell$-linetons will produce hook fields with a number of boxes in the first column with the parity of $r$, the tallest hook field having chirality left (resp. right). The product of a left (resp. right) Di $\ell$-lineton with a right (resp. left) Di $\ell$-lineton gives the hook fields with a number of boxes in the first column with the parity of $r+1$.

Remark. The proof of the above theorem amounts to decomposing a product of characters, namely rational functions of variables $q, x_{1}, \ldots, x_{r}$, into a sum of characters. The method of characters does not allow one to identify, in the aforementioned decomposition, whether the resulting characters are associated with decomposable or indecomposable modules. Since some of the representations considered here are non-unitary, they can be reducible but indecomposable, i.e. not fully reducible. Therefore, the symbols $\oplus$ appearing in the theorem should be understood in the weak sense, i.e. either direct or semi-direct sums. Only an explicit realisation of the modules can allow the precise distinction, for example in a holographic context where the various modules are realised as fields with prescribed boundary behaviours.

\section{Casimir energy}

Let us briefly recall the standard relations between characters of $\mathfrak{s o}(2, d)$, canonical partition functions of $C F T_{d}$ and the Casimir energy on $\mathbb{R} \times S^{d-1}$ (see e.g. [28, 33] for more explanations).

The canonical partition function $\mathcal{Z}(\beta)$ for a free boundary conformal field theory on $\mathbb{R} \times$ $S^{d-1}$ (or for a free field in the anti-holographic bulk dual) in a thermal bath of temperature $T=1 / \beta$ is equal to the corresponding $\mathfrak{s o}(2, d)$-character evaluated at $q=\exp (-\beta)$ and $x_{1}=\ldots=x_{r}=1$. The Hamiltonian zeta-function can be defined in terms of the Mellin transform of the canonical partition function

$$
\zeta_{E}(z):=\frac{1}{\Gamma(z)} \int_{0}^{\infty} d \beta \beta^{z-1} \mathcal{Z}(\beta),
$$

whose value at $z=-1$ is twice the Casimir energy $E_{C}=\frac{1}{2} \zeta_{E}(-1)$.

As pointed out in [28], the vanishing of the Casimir energy can sometimes be shown without computing explicitly the Hamitonian zeta-function due to the following property: when the canonical partition function is an even function of the temperature, $\mathcal{Z}(-\beta)=$ $\mathcal{Z}(\beta) \Leftrightarrow \mathcal{Z}(1 / q)=\mathcal{Z}(q)$, the Casimir energy automatically vanishes, $E_{C}=0$. Let us 
shortly review the heuristic reasoning behind this fact. As $\Gamma(z)$ has a pole at $z=-1$, the function $\frac{1}{\Gamma(z)}$ has a zero at $z=-1$, so for $\zeta_{E}(-1)$ to be non-zero, the integral has to have a pole at $z=-1$. The expansion in Laurent series around $\beta=0$ of an even function $\mathcal{Z}(\beta)$ of course only contains even powers of $\beta$. Thus, the integral in (5.1) can only have poles at even integer values of $z$. So the pole at $z=-1$ of the Gamma function at the denominator in (5.1) cannot be compensated by a pole from the integral. Consequently, the Hamiltonian zeta-function vanishes at $z=-1: E_{C}=\frac{1}{2} \zeta_{E}(-1)=0$.

As we will show below, the canonical partition functions for any product of two (scalar or spinor) higher-order singletons happen to be even in $\beta$, thereby implying the vanishing of the total Casimir energy for the infinite tower of fields displayed in our theorem. More rigorously, we have checked this in two ways: on the one hand, we have computed the Hamitonian zeta function for the square of the $\ell$-lineton partition function; on the other hand, we computed the infinite sum of the individual Casimir energies. Both ways give zero.

Using $\mathcal{P}^{(d)}(q, 1 \ldots, 1)=(1-q)^{-d}$, we have the following expression for the partition function of a single Rac $\ell$-lineton:

$$
\mathcal{Z}(q)=\chi_{\left[\frac{d}{2}-\ell, 0\right]}^{(d, 2)}(q, 1, \ldots, 1)=\frac{q^{\frac{d}{2}}\left(q^{-\ell}-q^{\ell}\right)}{(1-q)^{d}} .
$$

This partition function $\mathcal{Z}(\beta)$ is an even/odd function of $\beta$ when $d$ is odd/even:

$$
\mathcal{Z}\left(q^{-1}\right)=\frac{q^{-\frac{d}{2}}}{\left(1-q^{-1}\right)^{d}}\left(q^{\ell}-q^{-\ell}\right)=(-1)^{d+1} \mathcal{Z}(q) .
$$

The same parity property holds for the partition function of a single Di $\ell$-lineton. Therefore the partition functions for the tensor product of any two scalar or spinor higher-order singletons (possibly of distinct orders) are even functions of $\beta$, implying the vanishing of the corresponding Casimir energies. One can check that this is indeed the case by a direct calculation. Here we show it for two Rac $\ell$-lineton, the calculation being similar for the other cases.

Using $(1-x)^{-\alpha}=\sum_{n=0}^{+\infty}\left(\begin{array}{c}n+\alpha-1 \\ \alpha-1\end{array}\right) x^{n}$, one finds that the Hamiltonian zeta-function of the square of $\mathcal{Z}(\beta)$ given in (5.2) is equal to

$$
\begin{aligned}
\zeta_{E}(z) & =\frac{1}{\Gamma(z)} \int_{0}^{+\infty} d \beta \beta^{z-1} \frac{e^{-\beta d}\left(e^{2 \ell \beta}+e^{-2 \ell \beta}-2\right)}{\left(1-e^{-\beta}\right)^{2 d}} \\
& =\frac{1}{\Gamma(z)} \sum_{n=0}^{+\infty}\left(\begin{array}{c}
n+2 d-1 \\
2 d-1
\end{array}\right) \int_{0}^{+\infty} d \beta \beta^{z-1} e^{-\beta(d+n)}\left(e^{2 \ell \beta}+e^{-2 \ell \beta}-2\right) .
\end{aligned}
$$

Now using $\int_{0}^{+\infty} d \beta \beta^{z-1} e^{-a \beta}=a^{-z} \Gamma(z)$, we have :

$$
\zeta_{E}(z)=\sum_{n=0}^{+\infty}\left(\begin{array}{c}
n+2 d-1 \\
2 d-1
\end{array}\right)\left[(d+n-2 \ell)^{-z}+(d+n+2 \ell)^{-z}-2(d+n)^{-z}\right] .
$$

For $z=-1$, the sum of the three terms in square brackets vanishes for any fixed value of $n$. As a result, $E_{C}=0$.

Notice that, contrarily to the odd-dimensional case, the partition function of individual multi-linetons in even dimension $d$ are odd functions of $\beta$ and their Casimir energy are nonvanishing. Nevertheless, the Casimir energy vanishes for the tensor product of two of them. 


\section{Conclusions and perspectives}

In this note, we generalised the Flato-Fronsdal theorem to the cases of higher-order singletons, scalar and spinor, in arbitrary dimensions $d \geqslant 3$. We found novelties in the case of the tensor product of two spinor $\ell$-linetons, such as degeneracies and partially-massless mixed-symmetry fields of any depth. The tensor product of two scalar or spinor multilinetons of any two different orders has been decomposed as well. This result is presented in the appendix.

Our theorem in section 4 suggests the existence of a higher-spin, unbroken Vasilievlike theory that should be the bulk dual of a Gross-Neveu model at an isotropic Lifshitz fixed point. The spectrum of this conjectured higher-spin gravity theory is encoded in the decomposition of two Di $\ell$-linetons given in our theorem. A first check of this holographic conjecture and the one in [14] is the vanishing of the total Casimir energy at 1 loop for both spectra. These conjectures and their corresponding one-loop checks admit a straightforward generalisation for a direct sum of free scalar and spinor singletons of various orders.

An interesting generalisation of our results is to look at tensor products of higher-order spin- $s$ singletons for $s \geqslant 1$ (in even dimensions $d$ ). It would also be interesting to understand how the results obtained by the method of $\mathfrak{s o}(2, d)$ characters can be "continued" to the signature $\mathfrak{s o}(1, d+1)$. This is motivated by the unitarity of partially-massless fields in de Sitter geometries. For the same reason that the bosonic Vasiliev theory is unitary in de Sitter spacetime, the higher-spin gravity theories whose spectra we found in the present note should also be unitary in de Sitter background.

\section{Acknowledgments}

We are grateful to Ph. Spindel for various discussions. The research of X.B. was supported by the Russian Science Foundation grant 14-42-00047 in association with Lebedev Physical Institute. X.B. wants to thank the F.R.S.-FNRS (Belgium) and the Service de Mécanique et Gravitation for financial support and hospitality during the completion of this work. The work of N.B. was partially supported by a contract "Actions de Recherche concertées — Communauté française de Belgique” AUWB-2010-10/15-UMONS-1.

\section{A General theorem}

The tensor product of two higher-order singletons, scalar and spinor, decomposes as:

$$
\begin{aligned}
\mathcal{D}\left(\frac{d}{2}-\ell, \mathbf{0}\right) \otimes \mathcal{D}\left(\frac{d}{2}-\ell^{\prime}, \mathbf{0}\right) & \cong \bigoplus_{k=\frac{\mid \ell-\ell^{\prime}}{2}+1}^{\frac{\ell+\ell^{\prime}}{2}} \bigoplus_{s=0}^{\infty} \mathcal{D}(s+d-2 k, s, \mathbf{0}), \\
\mathcal{D}\left(\frac{d+1}{2}-\ell, \frac{\mathbf{1}}{\mathbf{2}}\right) \otimes \mathcal{D}\left(\frac{d}{2}-\ell^{\prime}, \mathbf{0}\right) & \cong \bigoplus_{t=\left|\ell-\ell^{\prime}-\frac{1}{2}\right|+\frac{1}{2}}^{\ell+\ell^{\prime}-1} \bigoplus_{s=\frac{1}{2}, \frac{3}{2}, \ldots}^{\infty} \mathcal{D}\left(s+d-t-1, s, \frac{\mathbf{1}}{\mathbf{2}}\right) .
\end{aligned}
$$


In the cases $d=2 r+1$, we have, for $\ell \neq \ell^{\prime}$ :

$$
\begin{aligned}
\mathcal{D}\left(\frac{d+1}{2}-\ell, \frac{\mathbf{1}}{\mathbf{2}}\right) \oplus \mathcal{D}\left(\frac{d+1}{2}-\ell^{\prime}, \frac{\mathbf{1}}{\mathbf{2}}\right) \cong \bigoplus_{t=\left|\ell-\ell^{\prime}\right|}^{\ell+\ell^{\prime}-2}[\mathcal{D}(d-1+t, \mathbf{0}) \oplus \mathcal{D}(d-1-t, \mathbf{0})] \\
\oplus \bigoplus_{s=1}^{\infty} \bigoplus_{m=0}^{r-1}\left[\bigoplus_{t=\left|\ell-\ell^{\prime}\right|+1}^{\ell+\ell^{\prime}-2} 2 \mathcal{D}\left(s+d-t-1, s, \mathbf{1}^{m}\right)\right. \\
\left.\oplus \mathcal{D}\left(s+d-\left|\ell-\ell^{\prime}\right|-1, s, \mathbf{1}^{m}\right) \oplus \mathcal{D}\left(s+d-\ell-\ell^{\prime}, s, \mathbf{1}^{m}\right)\right],
\end{aligned}
$$

while in the cases $d=2 r$ and for $\ell \neq \ell^{\prime}$ :

$$
\begin{aligned}
\mathcal{D}\left(\frac{d+1}{2}-\ell, \frac{\mathbf{1}}{\mathbf{2}}\right) \otimes \mathcal{D}\left(\frac{d+1}{2}-\ell, \frac{\mathbf{1}}{\mathbf{2}}\right) \cong \bigoplus_{t=\left|\ell-\ell^{\prime}\right|}^{\ell+\ell^{\prime}-2}[2 \mathcal{D}(d-1+t, \mathbf{0}) \oplus 2 \mathcal{D}(d-1-t, \mathbf{0})] \\
\oplus \bigoplus_{s=1}^{\infty} \bigoplus_{m=0}^{r-2}\left[\bigoplus_{t=\left|\ell-\ell^{\prime}\right|+1}^{\ell+\ell^{\prime}-2} 4 \mathcal{D}\left(s+d-t-1, s, \mathbf{1}^{m}\right)\right. \\
\left.\qquad 2 \mathcal{D}\left(s+d-\left|\ell-\ell^{\prime}\right|-1, s, \mathbf{1}^{m}\right) \oplus 2 \mathcal{D}\left(s+d-\ell-\ell^{\prime}, s, \mathbf{1}^{m}\right)\right] \\
\oplus \bigoplus_{s=1}^{\infty}\left[\bigoplus_{t=\left|\ell-\ell^{\prime}\right|+1}^{\ell+\ell^{\prime}-2}\left(2 \mathcal{D}\left(s+d-t-1, s, \mathbf{1}^{r-2}, 1\right) \oplus 2 \mathcal{D}\left(s+d-t-1, s, \mathbf{1}^{r-2},-1\right)\right)\right. \\
\left.\oplus \mathcal{D}\left(s+d-\left|\ell-\ell^{\prime}\right|-1, s, \mathbf{1}^{r-2}, 1\right) \oplus \mathcal{D}\left(s+d-\ell-\ell^{\prime}, s, \mathbf{1}^{r-2},-1\right)\right] .
\end{aligned}
$$

Open Access. This article is distributed under the terms of the Creative Commons Attribution License (CC-BY 4.0), which permits any use, distribution and reproduction in any medium, provided the original author(s) and source are credited.

\section{References}

[1] M. Flato and C. Fronsdal, One massless particle equals two Dirac singletons: elementary particles in a curved space. 6, Lett. Math. Phys. 2 (1978) 421 [INSPIRE].

[2] M.A. Vasiliev, Equations of motion of interacting massless fields of all spins as a free differential algebra, Phys. Lett. B 209 (1988) 491 [INSPIRE].

[3] M.A. Vasiliev, Consistent equation for interacting gauge fields of all spins in (3+1)-dimensions, Phys. Lett. B 243 (1990) 378 [inSPIRE].

[4] X. Bekaert, N. Boulanger and P. Sundell, How higher-spin gravity surpasses the spin two barrier: no-go theorems versus yes-go examples, Rev. Mod. Phys. 84 (2012) 987 [arXiv: 1007.0435] [INSPIRE].

[5] V.E. Didenko and E.D. Skvortsov, Elements of Vasiliev theory, arXiv:1401.2975 [INSPIRE]. 
[6] E. Sezgin and P. Sundell, Massless higher spins and holography, Nucl. Phys. B 644 (2002) 303 [Erratum ibid. B 660 (2003) 403] [hep-th/0205131] [INSPIRE].

[7] I.R. Klebanov and A.M. Polyakov, AdS dual of the critical $O(N)$ vector model, Phys. Lett. B 550 (2002) 213 [hep-th/0210114] [INSPIRE].

[8] E. Sezgin and P. Sundell, Holography in $4 D$ (super) higher spin theories and a test via cubic scalar couplings, JHEP 07 (2005) 044 [hep-th/0305040] [INSPIRE].

[9] P.A.M. Dirac, A remarkable representation of the $3+2$ de Sitter group, J. Math. Phys. 4 (1963) 901 [inSPIRE].

[10] W. Heidenreich, Tensor products of positive energy representations of $\mathrm{SO}(3,2)$ and $\mathrm{SO}(4,2)$, J. Math. Phys. 22 (1981) 1566 [INSPIRE].

[11] M.A. Vasiliev, Higher spin superalgebras in any dimension and their representations, JHEP 12 (2004) 046 [hep-th/0404124] [INSPIRE].

[12] F.A. Dolan, Character formulae and partition functions in higher dimensional conformal field theory, J. Math. Phys. 47 (2006) 062303 [hep-th/0508031] [INSPIRE].

[13] C. Iazeolla and P. Sundell, A fiber approach to harmonic analysis of unfolded higher-spin field equations, JHEP 10 (2008) 022 [arXiv:0806.1942] [INSPIRE].

[14] X. Bekaert and M. Grigoriev, Higher order singletons, partially massless fields and their boundary values in the ambient approach, Nucl. Phys. B 876 (2013) 667 [arXiv:1305.0162] [INSPIRE].

[15] S. Deser and R.I. Nepomechie, Anomalous propagation of gauge fields in conformally flat spaces, Phys. Lett. B 132 (1983) 321 [INSPIRE].

[16] S. Deser and R.I. Nepomechie, Gauge invariance versus masslessness in de Sitter space, Annals Phys. 154 (1984) 396 [InSPIRE].

[17] S. Deser and A. Waldron, Partial masslessness of higher spins in (A)dS, Nucl. Phys. B 607 (2001) 577 [hep-th/0103198] [INSPIRE].

[18] E.D. Skvortsov and M.A. Vasiliev, Geometric formulation for partially massless fields, Nucl. Phys. B 756 (2006) 117 [hep-th/0601095] [INSPIRE].

[19] L. Dolan, C.R. Nappi and E. Witten, Conformal operators for partially massless states, JHEP 10 (2001) 016 [hep-th/0109096] [INSPIRE].

[20] S. Deser and A. Waldron, Null propagation of partially massless higher spins in (A)dS and cosmological constant speculations, Phys. Lett. B 513 (2001) 137 [hep-th/0105181] [INSPIRE].

[21] X. Bekaert and M. Grigoriev, Higher-order singletons and partially massless fields, Bulg. J. Phys. 41 (2014) 172.

[22] N. Boulanger, C. Iazeolla and P. Sundell, Unfolding mixed-symmetry fields in AdS and the BMV conjecture: I. General formalism, JHEP 07 (2009) 013 [arXiv:0812.3615] [INSPIRE].

[23] N. Boulanger, C. Iazeolla and P. Sundell, Unfolding mixed-symmetry fields in AdS and the BMV conjecture. II. Oscillator realization, JHEP 07 (2009) 014 [arXiv: 0812.4438] [INSPIRE].

[24] N. Boulanger and P. Sundell, An action principle for Vasiliev's four-dimensional higher-spin gravity, J. Phys. A 44 (2011) 495402 [arXiv:1102.2219] [INSPIRE]. 
[25] N. Boulanger, N. Colombo and P. Sundell, A minimal BV action for Vasiliev's four-dimensional higher spin gravity, JHEP 10 (2012) 043 [arXiv: 1205.3339] [INSPIRE].

[26] S. Giombi and I.R. Klebanov, One loop tests of higher spin AdS/CFT, JHEP 12 (2013) 068 [arXiv: 1308.2337] [INSPIRE].

[27] S. Giombi, I.R. Klebanov and B.R. Safdi, Higher spin $A d S_{d+1} / C F T_{d}$ at one loop, Phys. Rev. D 89 (2014) 084004 [arXiv: 1401.0825] [InSPIRE].

[28] S. Giombi, I.R. Klebanov and A.A. Tseytlin, Partition functions and casimir energies in higher spin $A d S_{d+1} / C F T_{d}$, Phys. Rev. D 90 (2014) 024048 [arXiv:1402.5396] [INSPIRE].

[29] O.V. Shaynkman, I.Y. Tipunin and M.A. Vasiliev, Unfolded form of conformal equations in $M$ dimensions and $o(M+2)$ modules, Rev. Math. Phys. 18 (2006) 823 [hep-th/0401086] [INSPIRE].

[30] J. Engquist and P. Sundell, Brane partons and singleton strings, Nucl. Phys. B 752 (2006) 206 [hep-th/0508124] [INSPIRE].

[31] J. Engquist, P. Sundell and L. Tamassia, On singleton composites in non-compact WZW models, JHEP 02 (2007) 097 [hep-th/0701051] [INSPIRE].

[32] R.R. Metsaev, Massless mixed symmetry bosonic free fields in d-dimensional Anti-de Sitter space-time, Phys. Lett. B 354 (1995) 78 [INSPIRE].

[33] G.W. Gibbons, M.J. Perry and C.N. Pope, Partition functions, the Bekenstein bound and temperature inversion in Anti-de Sitter space and its conformal boundary,

Phys. Rev. D 74 (2006) 084009 [hep-th/0606186] [INSPIRE]. 